\title{
Erratum to: TRAIL signaling is mediated by DR4 in pancreatic tumor cells despite the expression of functional DR5
}

\author{
Johannes Lemke • Andreas Noack • Dieter Adam • Vladimir Tchikov • Uwe Bertsch • \\ Christian Röder • Stefan Schütze • Harald Wajant • Holger Kalthoff • Anna Trauzold
}

Published online: 24 February 2011

(C) Springer-Verlag 2011

\section{Erratum to: J Mol Med}

\section{DOI 10.1007/s00109-010-0619-0}

The original version of this article appeared in $J \mathrm{Mol} \mathrm{Med}$ 88(7):729-740

The authors have informed the Editors that there turns out to be some confusion about the identity of one of the three pancreatic tumor cell lines used in their study. A recently and carefully performed Short Tandem Repeats (STR) analysis of the pancreatic tumor cell lines used in their laboratory revealed that the batch of the pancreatic cancer cell line PT45 analyzed in this study is genetically identical to the wellknown mammary cancer cell line MDA-MB-231 (the results of the STR analysis can be provided by the authors upon request). Thus, the experimental results on the presumptive "PT45" cell line have to be regarded in terms of this limitation.

Nonetheless, this error does not affect the general message and conclusions of this study since the other two pancreatic tumor cell lines investigated (Panc89 and Colo357) clearly demonstrate the findings reported by the authors. The authors confirm that the identity of these two cell lines was verified using the same STR analysis technique. Moreover, a subsequent article which appeared online in October 2010 (Stadel et al., 2010, Clin Cancer Res 16:5734-5749) came to the same conclusion.

The authors deeply regret this mistake and the confusion it might cause, since these "PT45" cells may also have been used by other groups.

The online version of the original article can be found at http://dx.doi. org/10.1007/s00109-010-0619-0.

\footnotetext{
J. Lemke $\cdot$ A. Noack $\cdot$ C. Röder $\cdot$ H. Kalthoff $\cdot$ A. Trauzold $(\triangle)$

Division of Molecular Oncology, Institute of Experimental Cancer

Research, Comprehensive Cancer Center North, UK S-H,

Arnold-Heller Str. 3, Haus 17,

24105 Kiel, Germany

e-mail: atrauzold@email.uni-kiel.de

D. Adam $\cdot$ V. Tchikov $\cdot$ U. Bertsch $\cdot$ S. Schütze

Institute of Immunology, UK S-H,

Kiel, Germany

H. Wajant

Division of Molecular Internal Medicine, Department of Internal

Medicine II, University Hospital Würzburg,

Röntgenring 11,

97070 Würzburg, Germany
} 